Изв. Крымск. Астрофиз. Обсерв. 117, № 1, 48-55 (2021) doi:10.31059/izcrao-vol117-iss1-pp48-55

УДК 524.387

\title{
Определение параметров слабоаккрецирующего поляра V379 Vir
}

\author{
М.В. Сусликов ${ }^{1}$, А.И. Колбин ${ }^{1,2}$ \\ 1 Казанский (Приволжский) федеральный университет, Казань, 420008, Россия \\ mvsuslikov@outlook.com \\ 2 Специальная астрофизическая обсерватория РАН, Нижний Архыз, 369167, Россия \\ kolbinalexander@mail.ru
}

Поступила в редакцию 26 октября 2021 г.

Аннотация. В настоящей работе выполнена оценка параметров поляра с низким темпом аккреции V379 Vir. Используя данные наземных и космических обсерваторий, нами было получено спектральное распределение энергии в широком спектральном диапазоне, исправленное за межзвездное поглощение. Его моделирование позволило определить массу и эффективную температуру белого карлика: $M_{1}=0.628 \pm 0.009 \mathrm{M}_{\odot}, T_{\text {eff }}=11250 \pm 70 \mathrm{~K}$. На основе полуамплитуды лучевых скоростей облучаемой поверхности вторичного компонента сделана оценка его массы $M_{2} \approx 0.027 \mathrm{M}_{\odot}$, а также наклонения орбитальной плоскости $i=50 \pm 5^{\circ}$.

Ключевые слова: катаклизмические переменные, новые, звезды: индивидуальные: V379 Vir

\section{1 Введение}

Поляры (переменные типа AM Her) представляют собой тесные двойные системы, состоящие из белого карлика с сильным магнитным полем (первичный компонент, $B \sim 10^{7}-10^{8}$ МГс) и холодного карлика главной последовательности, заполняющего свою полость Роша (вторичный компонент). Вещество вторичного компонента вытекает из окрестностей точки Лагранжа $L_{1}$ в полость Роша первичного компонента и изначально движется вдоль траектории, близкой к баллистической. Сильное магнитное поле белого карлика не дает возможности сформироваться аккреционному диску. После достижения альфвеновского радиуса главного компонента, где гидродинамическое давление аккреционной струи оказывается равным давлению магнитного поля $\left(\rho v^{2}=B^{2} / 8 \pi\right)$, ионизованное вещество захватывается магнитным полем и движется вдоль силовых линий в направлении магнитных полюсов. Аккрецируемый газ со сверхзвуковой скоростью выпадает на поверхность белого карлика и образует ударный фронт на высотах 0.01-0.1 радиуса аккретора. За ударным фронтом газ разогревается до высоких температур ( $T \sim 10-50$ кэВ) и, охлаждаясь, оседает на поверхность белого карлика, формируя так называемую аккреционную колонну. При этом основными механизмами охлаждения являются тормозное рентгеновское излучение и циклотронное излучение в оптическом диапазоне.

Для понимания происхождения и особенностей эволюции поляров важно определение физических параметров их компонентов. Однако решение данной задачи имеет множество трудностей. При высоких темпах аккреции подавляющий вклад в интегральное излучение системы вносит аккреционная струя и аккреционная колонна, что делает практически невозможным оценку параметров системы на основе оптических наблюдений. Лучшие условия достигаются при переходе системы в состояние с низкой аккрецией. Однако даже в случае видимости компонентов системы в спектре определение их параметров представляет собой трудную задачу. Так, линии белого карлика подвержены действию квадратичного эффекта Зеемана, препятствующего измерению его лучевых скоростей. На лучевые скорости вторичного компонента оказывает сильное влияние неоднородность распределения яркости по звездной поверхности. Зачастую орбитальное движение вторичного ком- 
понента проявляется лишь в эмиссиях, формируемых в облучаемой рентгеновским излучением поверхности звезды.

В данной работе мы выполнили определение параметров низкоаккрецирующего поляра V379 Vir. Первые исследования этой системы, выполненные Schmidt et al. (2005), отождествили ее с взаимодействующей короткопериодической переменной с магнитным белым карликом и коричневым карликом. Авторами была получена оценка среднего значения магнитного поля белого карлика 7 МГс, а также обнаружена периодическая переменность лучевой скорости эмиссии $\mathrm{H}_{\alpha}(P \approx 90$ мин $)$, образуемой в области переизлучения на вторичном компоненте. Обнаружение Burleigh et al. (2006) фотометрической переменности с таким же периодом, но находящейся в противофазе с кривой лучевых скоростей линии $\mathrm{H}_{\alpha}$, указало на наличие ударной области на поверхности белого карлика. В последующей работе Debes et al. (2006) было установлено наличие избыточного излучения в ближнем инфракрасном диапазоне, что указывает на присутствие холодного коричневого карлика спектрального класса L5-L7 с температурой фотосферы менее $1700 \mathrm{~K}$. Кривая блеска в $K_{s}$-полосе демонстрирует переменность с периодом $\approx 88$ мин, вызванную циклотронным излучением ударной области на поверхности белого карлика. Дальнейшие спектральные исследования в ближнем инфракрасном диапазоне, выполненные Farihi et al. (2008), подтвердили наличие коричневого карлика позднего L-класса. Анализ наблюдений XMM-Newton в рентгеновской области (Stelzer et al., 2017) выявил слабый темп аккреции около $10^{-14} \mathrm{M}_{\odot} /$ год. В данной работе мы выполнили моделирование спектрального распределения энергии V379 Vir с определением параметров белого карлика. Кроме того, на основе кривой лучевых скоростей области переизлучения, мы сделали оценку параметров вторичного компонента и наклонения орбитальной плоскости.

\section{2 Распределение плотности потока V379 Vir}

Фотометрические наблюдения V379 Vir выполнялись на различных наземных и космических обсерваториях в широком диапазоне длин волн. Наблюдения в видимом и ближнем инфракрасном диапазонах были получены в рамках обзора SDSS (Szkody et al., 2011), а также на телескопе VISTA (Edge et al., 2013). Потоки в ультрафиолетовом диапазоне были заимствованы из архивов наблюдений космических телескопов Swift и GALEX.

При построении спектрального распределения энергии V379 Vir учитывалось влияние межзвездного поглощения. Известно, что видимая звездная величина объекта оказывается ослабленной при прохождении излучения через межзвездную среду в результате поглощения и рассеяния. Характер зависимости поглощения от длины волны $A(\lambda)$ оказывает наибольшее ослабление излучения в коротковолновой области, что приводит к покраснению источника (Trumpler, 1930). Величину покраснения принято выражать через избыток цвета $E(B-V)$. Межзвездная экстинкция в фотометрической полосе $x$ и избыток цвета связаны между собой известным соотношением

$$
R_{x}=\frac{A_{x}}{E(B-V)},
$$

где $R_{x}$ - коэффициент экстинкции полосы пропускания $x$.

Для определения $E(B-V)$ исследуемого объекта использовалась связь этого параметра с колонковой плотностью нейтрального водорода на луче зрения. В своей работе Bohlin et al. (1978) изучали поглощение межзвездного нейтрального водорода в Галактике вблизи спектральной линии $\mathrm{L}_{\alpha}$. Они показали, что среднее отношение между колонковой плотностью НІ и избытком цвета $E(B-V)$ постоянно:

$$
\left\langle\frac{N_{\mathrm{HI}}}{E(B-V)}\right\rangle=4.8 \times 10^{21} \text { atoms cm}{ }^{-2} \mathrm{mag}^{-1} .
$$

B статье Stelzer et al. (2017) на основе моделирования рентгеновских данных V379 Vir была получена колонковая плотность нейтрального водорода на луче зрения $N_{H I}=2.3 \times 10^{20}$ атомов см ${ }^{-2}$. Используя это значение, согласно формуле (2) получена величина избытка цвета для V379 Vir $E(B-V)=0.048$. Коэффициенты экстинкции для фотометрических систем SDSS и VISTA были определены в работах Yuan et al. (2013) и Gonzalez-Fernandez et al. (2018) соответственно. Используя эти значения и вычисленный ранее избыток цвета $E(B-V)$ в уравнении $(1)$, нами получены поправки $A_{x}$, учитывающие межзвездное поглощение в оптике для звездных величин SDSS и VISTA. 
Данные наблюдений V379 Vir с обсерваторий Swift и GALEX в ультрафиолетовом диапазоне уже учитывают межзвездное поглощение.

Определение части параметров выполнялось на основе метода синтетической фотометрии. Поскольку основной вклад в излучение поляра в низком состоянии дает главный и вторичный компонент, то распределение плотности потока $f_{\nu}$ системы находилось сложением спектров белого и коричневого карликов. Для белого карлика спектральное распределение потока вычислялось на основе стационарных плоско-параллельных ЛТР-моделей звездных атмосфер, рассчитанных Koester (2010). Зафиксировав ускорение силы тяжести белого карлика на значении $\log g=8.0$, оцененном по фотометрическим наблюдениям (Schmidt et al., 2005), была получена сетка спектров для набора эффективных температур $T_{\text {eff }}$ Учет вторичного компонента проводился путем добавления к спектру белого карлика спектра звезды 2MASS J16322911+1904407 спектрального класса L8. Выбор этого спектрального класса был обусловлен его наилучшей аппроксимацией наблюдаемых инфракрасных спектров, выполненной Farihi et al. (2008).

Для описания наблюдаемого распределения спектральной плотности потока использовался взвешенный метод наименьших квадратов, заключающийся в минимизации функции

$$
\chi^{2}\left(\theta, T_{\text {eff }}\right)=\sum_{k}^{l}\left(\frac{\theta^{2} f_{k}\left(T_{\text {eff }}\right)-f_{k}^{\text {obs }}}{\sigma_{k}}\right)^{2},
$$

где $\theta$ - угловой радиус источника в радианах, $f_{k}$ и $f_{k}^{\text {obs }}$ - теоретический и наблюдаемый поток соответственно, $\sigma_{k}$ - ошибки фотометрии, а индекс $k$ соответствует фотометрической полосе. В процессе минимизации выражения (3) находился угловой радиус белого карлика, а также его эффективная температура.

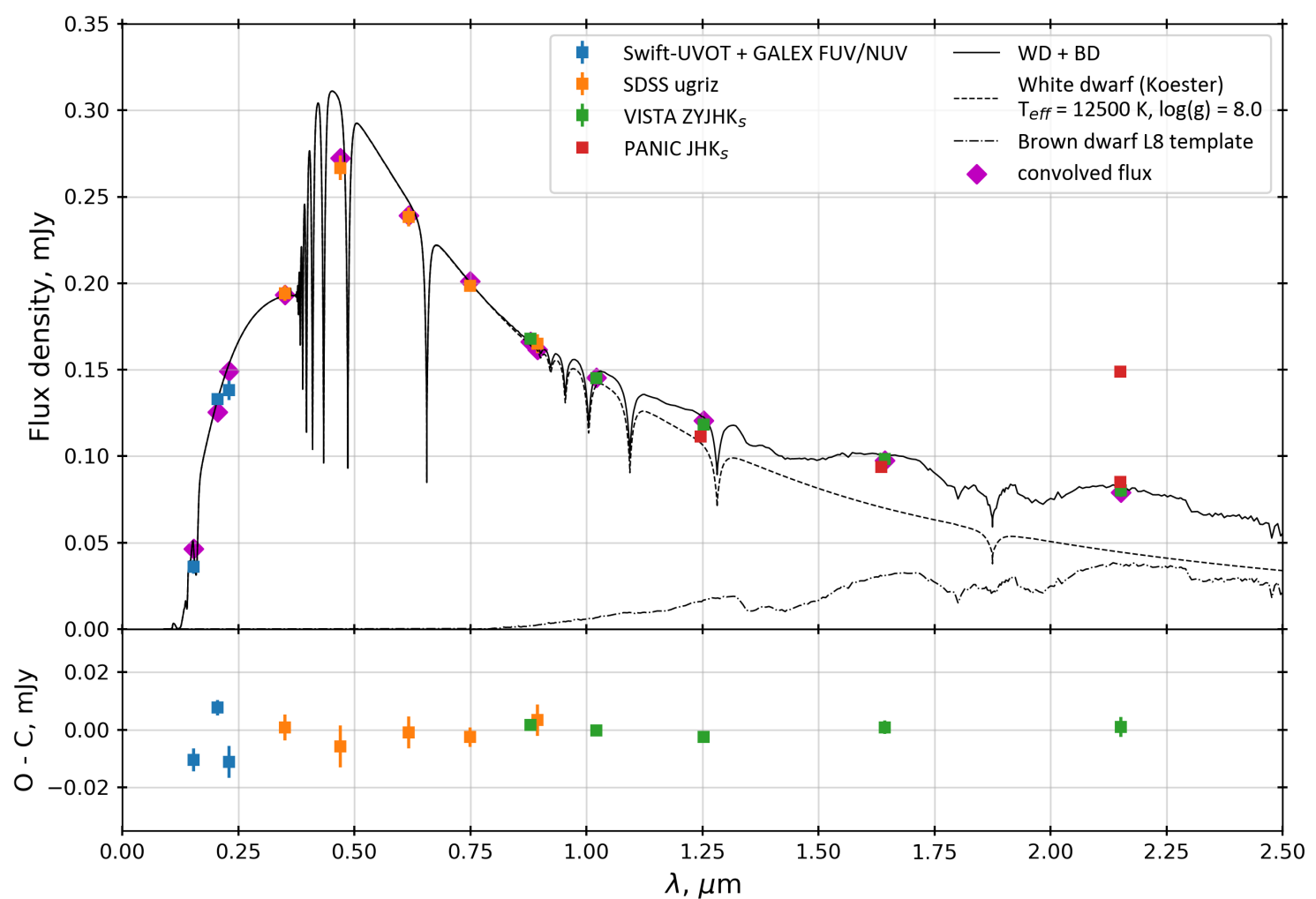

Рис. 1. Распределение спектральной плотности потока V379 Vir

Поскольку в визуальной и ультрафиолетовой области спектра исследуемой системы доминирует излучение главного компонента, для более точного определения его параметров выполнялась аппроксимация модельным спектром белого карлика в фотометрических потоках Swift-UVOT, GALEX и SDSS. Результат описания наблюдаемого распределения спектральной плотности потока моделью 
белого карлика представлен пунктирной линией на рис. 1. Подобранная модель спектра белого карлика имеет температуру $T_{\text {eff }}=11250 \pm 70 \mathrm{~K}$. Также был получен угловой радиус главной компоненты $\theta=3.754 \times 10^{-7} \pm 0.032 \times 10^{-7 \prime \prime}$. Чтобы выполнить аппроксимацию инфракрасных потоков VISTA к модели белого карлика был добавлен спектр коричневого карлика L8. Итоговый интегральный спектр представлен на рис. 1 сплошной линией. Отклонение наблюдаемых потоков от синтетических по $\mathrm{O}$ - С-диаграмме составляет менее $0.2 \mathrm{мЯн,} \mathrm{что} \mathrm{говорит} \mathrm{о} \mathrm{хорошем} \mathrm{согласовании} \mathrm{двухкомпонент-}$ ной модели с наблюдениями. На график были нанесены усредненные потоки из кривых блеска в полосах $\mathrm{JHK}_{s}$, полученных Debes et al. (2006). Для них также было учтено межзвездное поглощение. Из кривой блеска $\mathrm{K}_{s}$ нами было получено два значения плотности потока: при усреднении звездных величин в интервале фаз $\Delta \phi=0.8-1.2$ с постоянным излучением от вторичного компонента и в интервале $\Delta \phi=0.2-0.8$, соответствующем появлению источника циклотронного излучения. Для оценки ошибок определения параметров, найденных из описания наблюдаемого спектрального распределения энергии, применялся метод Монте-Карло.

\section{3 Определение параметров компонент}

По полученному ранее угловому радиусу главного компонента и параллаксу V379 Vir $\pi=6.68 \times$ $10^{-3} \pm 0.18 \times 10^{-3 \prime \prime}$, заимствованному из каталога GAIA DR3 (Gaia Collaboration, 2018), был определен радиус белого карлика $R_{1}=0.0121 \pm 0.0001 \mathrm{R}_{\odot}$. Как известно, для белых карликов существует зависимость их радиуса от массы, которая была выражена Nauenberg (1972) в аналитическом виде:

$$
R_{1}=7.795 \times 10^{8}\left[\left(\frac{1.44 M_{\odot}}{M_{1}}\right)^{2 / 3}-\left(\frac{M_{1}}{1.44 M_{\odot}}\right)^{2 / 3}\right]^{1 / 2} \mathrm{~cm} .
$$

Решение этого уравнения дает оценку массы белого карлика $M_{1}=0.628 \pm 0.009 \mathrm{M}_{\odot}$. Соответствующее ускорение силы тяжести у поверхности главного компонента равно $\log g=8.07$, что хорошо согласуется с оценкой этого параметра, полученной в работе Schmidt et al. $(2005)(\log g=8.0)$.

В выполненном Schmidt et al. (2005) спектральном исследовании V379 Vir получена кривая лучевых скоростей эмиссии линии $\mathrm{H}_{\alpha}$. Считается, что источником этой эмиссии является поверхность вторичного компонента, облучаемая рентгеновским излучением аккреционного пятна. Авторами была получена оценка полуамплитуды кривой лучевых скоростей данной области $K_{2}^{\prime}=320 \pm 20$ км$/$ c. Однако для оценки параметров звезды необходимо найти полуамплитуду лучевых скоростей центра масс $K_{2}$. Полуамплитуда $K_{2}$ была найдена нами путем моделирования облучаемой рентгеновским излучением атмосферы холодной звезды, обращенной в сторону аккретора (cм. Shimanskii et al., 2012). В процессе моделирования предполагалась синхронность вращения вторичного компонента с его орбитальным движением. Была принята рентгеновская светимость аккреционного пятна $L_{X}=3 \times 10^{29}$ эрг/c (Stelzer et al., 2017), а форма спектрального распределения в рентгеновской области выбрана согласно данным Cropper (1990). Теоретические лучевые скорости эмиссии Н $\alpha$ измерялись методом кросс-корреляции в 72 фазах орбитального периода. На основе этих измерений вычислена поправка к полуамплитуде лучевой скорости $\Delta K_{2}=K_{2}-K_{2}^{\prime}=26.8 \pm 1.3 \mathrm{~km} / \mathrm{c}$.

Используя фотометрические наблюдения Debes et al. (2006), нами был уточнен орбитальный период системы $P_{\text {orb }}=0 .^{d} 060781$ с применением метода Лафлера-Кинмана. Функция масс $f\left(M_{2}\right)$ была вычислена согласно формуле

$$
f\left(M_{2}\right)=1.0385 \times 10^{-7} P_{\text {orb }} K_{2}^{3}
$$

и оказалась равной $f\left(M_{2}\right)=0.263 \pm 0.049 M_{\odot}$. С другой стороны, известно, что

$$
f\left(M_{2}\right)=\frac{M_{1}^{3}}{\left(M_{1}+M_{2}\right)^{2}} \sin ^{3} i,
$$

где $M_{1}$ - масса белого карлика, $M_{2}$ - масса вторичного компонента, а $i$ - наклонение орбитальной плоскости.

Дополнительные ограничения, необходимые для определения параметров компонентов V379 Vir, можно получить из предположения близости вторичного компонента к заполнению своей полости 
Роша. Согласно Eggleton (1983), эффективный радиус $R_{L}$ полости Роша вторичного компонента может быть оценен как

$$
\frac{R_{L}}{A}=0.49 \frac{q^{2 / 3}}{0.6 q^{2 / 3}+\ln \left(1+q^{1 / 3}\right)}
$$

где $q=M_{2} / M_{1}$ - отношение масс вторичного и главного компонентов, $A$ - разделение центров масс компонентов, для определения которого можно воспользоваться третьим законом Кеплера: $A=$ $\left(M_{1}(1+q) P_{\mathrm{orb}}^{2}\right)^{1 / 3}$.

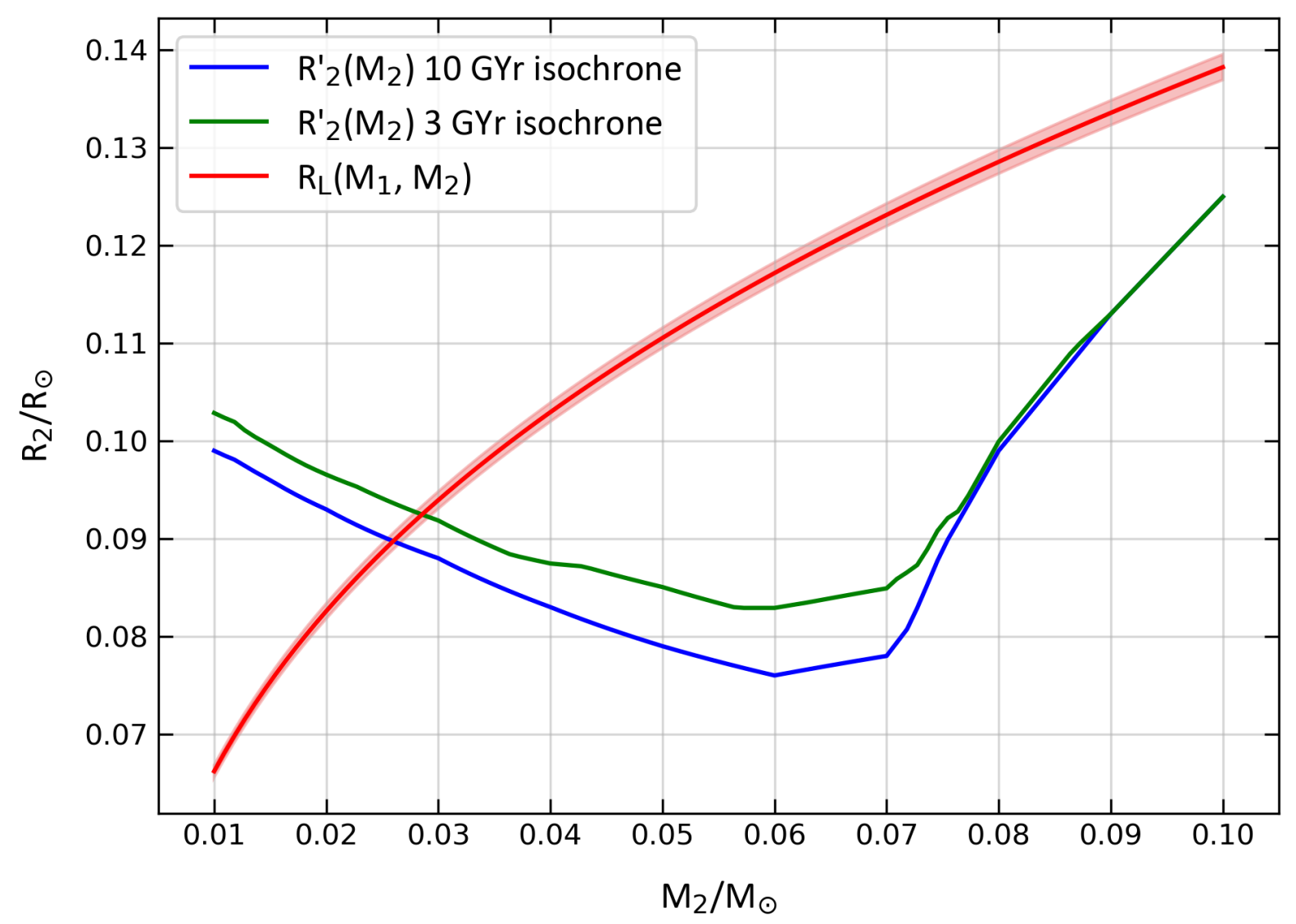

Рис. 2. Эволюционные зависимости "Радиус - Масса" для коричневых карликов, соответствующие возрастам $3 \times 10^{9}$ и $10^{10}$ лет (зеленая и синяя линии соответственно), а также эффективный радиус полости Роша вторичного компонента (красная линия)

В то же время радиус вторичного компонента связан с его массой эволюционной зависимостью $R_{2}^{\prime}\left(M_{2}\right)$. Эта зависимость была получена из изохрон Baraffe et al. (2003) для солнечного содержания элементов $(Z=0.019)$. Ограничив возраст системы между $t_{1}=3 \times 10^{9}$ и $t_{2}=10^{10}$ лет и решая уравнение $R_{2}^{\prime}\left(M_{2}\right)=R_{L}\left(M_{2}, M_{1}\right)$ для двух указанных возрастов, мы нашли массу вторичного компонента $M_{2}=0.027 \pm 0.001 M_{\odot}(\approx 28.5$ масс Юпитера $)$ и его эффективный радиус $R_{2} \approx 0.091 R_{\odot}$. Графическое представление эволюционных зависимостей $R_{2}^{\prime}\left(M_{2}\right)$ и эффективного радиуса полости Роша $R_{L}\left(M_{2}, M_{1}\right)$ для $M_{1}=0.628 \pm 0.009 M_{\odot}$ дано на рис. 2 . Подстановка найденных масс компонентов в выражение (6) дает наклонение орбитальной плоскости $i=50 \pm 5^{\circ}$. Из третьего закона Кеплера получено разделение компонентов $A=0.565 \pm 0.003 R_{\odot}$. Решая систему уравнений функции масс главного компонента $f\left(M_{1}\right)$, находим полуамплитуду кривой лучевых скоростей белого карлика $K_{1}=15.1 \pm 0.7 \mathrm{kм} / \mathrm{c}$. Все найденные параметры с ошибками их определения перечислены в таблице 1 . 
Таблица 1. Параметры системы V379 Vir

\begin{tabular}{lcc}
\hline Параметр & Значение & Ошибка \\
\hline$T_{\mathrm{eff}}, \mathrm{K}$ & 11250 & 70 \\
$\log g$ & 8.07 & \\
$P_{\mathrm{orb}}, \mathrm{cyт}$ & 0.060781 & \\
$\pi, \times 10^{-3}{ }^{\prime \prime}$ & 6.68 & 0.18 \\
$d$, пк & 149 & 4 \\
$i,{ }^{\circ}$ & 50 & 5 \\
$K_{1}, \mathrm{KM} / \mathrm{c}$ & 15.1 & 0.7 \\
$K_{2}, \mathrm{KM} / \mathrm{c}$ & 346.8 & 21.3 \\
$R_{1}, R_{\odot}$ & 0.0121 & 0.0001 \\
$R_{2}, R_{\odot}$ & 0.091 & 0.001 \\
$M_{1}, M_{\odot}$ & 0.628 & 0.009 \\
$M_{2}, M_{\odot}$ & 0.027 & 0.001 \\
$A, R_{\odot}$ & 0.565 & 0.003 \\
\hline
\end{tabular}

\section{4 Заключение}

В данной работе выполнено фотометрическое исследование поляра V379 Vir, находящегося в режиме низкого темпа аккреции. Используя результаты наблюдений на различных наземных и космических обсерваториях в широком диапазоне длин волн, удалось построить спектральное распределение энергии V379 Vir, исправленное за межзвездное поглощение. Путем описания наблюдаемых потоков комбинацией спектров белого карлика и коричневого карлика спектрального класса L8 была получена оценка температуры главного компонента $T_{\text {eff }}=11250 \pm 70 \mathrm{~K}$. По известному параллаксу GAIA и угловому размеру главного компонента были определены его радиус $R_{1}=0.0120 \pm 0.0001 \mathrm{R}_{\odot}$ и масса $M_{1}=0.628 \pm 0.009 M_{\odot}$. Используя полуамплитуду кривой лучевых скоростей области переизлучения на вторичном компоненте $K_{2}^{\prime}$, а также предположив заполнение донором своей полости Роша, нами получена оценка наклонения орбитальной плоскости $i=50 \pm 5^{\circ}$, а также масса и радиус вторичного компонента $M_{2} \approx 0.027 M_{\odot}$ и $R_{2} \approx 0.091 R_{\odot}$ соответственно.

Благодарности. Исследование выполнено при финансовой поддержке РФФИ в рамках научного проекта № 19-32-60048.

\section{Литература}

Baraffe I., Chabrier G., Barman T.S., et al., 2003. Astron. Astrophys., vol. 402, pp. 701-712.

Bohlin R.C., Savage B.D., Drake J.F., 1978. Astrophys. J., vol. 224, pp. 132-142.

Burleigh M.R., Marsh T.R., Gansicke B.T., et al., 2006. Mon. Not. Roy. Astron. Soc., vol. 373, pp. 14161422.

Cropper M., 1990. Space Sci. Rev., vol. 54, pp. 195-295.

Debes J.H., Lopez-Morales M., Bonanos A.Z., et al., 2006. Astrophys. J., vol. 647, pp. 147-150.

Edge A., Sutherland W., Viking Team, 2013. VizieR On-line Data Catalog: II/343.

Eggleton P.P., 1983. Astrophys. J., vol. 268, pp. 368-369.

Farihi J., Burleigh M.R., Hoard D.W., 2008. Astrophys. J., vol. 674, pp. 421-430.

Gaia Collaboration, 2018. VizieR On-line Data Catalog: I/345.

Girardi L., Bressan A., Bertelli G., et al., 2000. Astron. Astrophys. Suppl. Ser., vol. 141. pp. 371-383.

Gonzalez-Fernandez C., Hodgkin S.T., Irwin M.J., et al., 2018. Mon. Not. Roy. Astron. Soc., vol. 474, pp. 5459-5478.

Koester D., 2010. Memorie della Societa Astronomica Italiana, vol. 81. pp. 921-931.

Nauenberg M., 1972. Astrophys. J., vol. 175, p. 417. 
Schmidt G.D., Szkody P., Silvestri N.M., et al., 2005. Astrophys. J., vol. 630, pp. 173-176.

Shimanskii V.V., Karitskaya E.A., Bochkarev N.G., et al., 2012. Astron. Rep, vol. 56, pp. 741-760.

Stelzer B., de Martino D., Casewell S.L., et al., 2017. Astron. Astrophys., vol. 598, p. 6.

Szkody P., Anderson S.F., Brooks K., et al., 2011. Astron. J., vol. 142, p. 9.

Trumpler R.J., 1930. Lick Observatory bulletin, vol. 420. pp. 154-188.

Yuan H.B., Liu X.W., Xiang M.S., 2013. Mon. Not. Roy. Astron. Soc., vol. 430, pp. 2188-2199. 


\title{
Determination of the parameters of the low accretion rate polar V379 Vir
}

\author{
M.V. Suslikov ${ }^{1}$, A.I. Kolbin ${ }^{1,2}$
}

1 Kazan (Volga Region) Federal University, Kremlyovskaya st. 18, Kazan 420008, Russian Federation mvsuslikov@outlook.com

${ }^{2}$ Special Astrophysical Observatory of Russian Academy of Sciences, Nizhnij Arkhyz 369167, Karachai-Cherkessian Republic, Russian Federation kolbinalexander@mail.ru

Abstract. This study was carried out to determine the parameters of the low accretion polar V379 Vir. We have obtained the broad-band spectral energy distribution (SED) corrected for interstellar extinction due to the data from various ground-based and space observatories. According to the SED approximation by the model of a white dwarf + brown dwarf L8, the mass and effective temperature of the white dwarf are $M_{1}=0.628 \pm 0.009 \mathrm{M}_{\odot}, T_{\text {eff }}=11250 \pm 70 \mathrm{~K}$. The estimations of donor mass $M_{2} \approx 0.027 \mathrm{M}_{\odot}$ and orbital inclination $i=50 \pm 5^{\circ}$ were obtained based on the radial velocity semi-amplitude of the irradiated secondary hemisphere.

Key words: cataclysmic variables, novae, stars: individual: V379 Vir 